\title{
Mean curvatures of a subspace in a Finsler space.
}

\author{
Memoria di M. Y. Zhang (a Chekiang, Cina).
}

Sunto. - Il contenuto del presente lavoro trovasi riassunto nel seguente capoverso.

In a recent paper (1) the author has generalized CALONGHX's theorem ("), concerning the mean curvature of a surface in ordinary space, to a threedimensional FinsLer space. The geometrical definition of the mean curvature thus obtained is independent of the choice of the element of support of the space. The object of the present paper is to give an extension to an $m$-dimensional subspace $F_{m}$ of an $n$-dimensional FinsLer space $F_{n}$. For clearness, we shall begin by investigating the mean curvature of a hypersurface, and then consider the general case.

1. The mean eurvature of a hypersurface $F_{n-1}$ in $F_{n}$.

Let $F_{n}$ be an $n$-dimensional FinsLer space with the element of support $\left(x, x^{\prime}\right)$, along which the contravariant components of the unit vector are denoted by $l^{i}(i=1,2, \ldots, n)$. Let $g_{i k}\left(x, x^{\prime}\right)(i, k=1,2, \ldots, n)$ be the covariant components of the metric tensor. We consider a hypersurface $F_{n-1}$ of equations

$$
x^{i}=x^{i}\left(u^{\lambda}\right):
$$

hereafter Latin indices $i, j, k, \ldots$ are in the range $1,2, \ldots, n$ and Greek indices $\lambda, \mu, \nu, \ldots$ in the range $1,2, \ldots, n-1$. For a tensor in $F_{n-1}$ of components $T_{\mu}^{\lambda}$, the components in $F_{n}$ are given by the equations

where

$$
T_{j}^{i}=T_{\mu}^{\lambda} \gamma_{\lambda}^{i} \vartheta_{j}^{\mu},
$$

$$
\vartheta_{\lambda}^{i}=\frac{\partial x^{i}}{\partial u^{\lambda}}, \quad \vartheta_{j}^{\mu}=g_{i k} g^{\lambda \mu \vartheta_{\lambda}^{k}}
$$

We assume that all the functions throughout the following discussion are analytic.

(i) ZIIANG MiNG $\mathrm{X}_{\mathrm{NG}}$, Die mittlere Krummung einer Fläche im dreidimensionalen Finslerschen Raum, "Science Record, Academia Sinica „, new series, vol. 1 (1950), n. 1.

${ }_{(2)}$ M. CALonghi, Sulla curvatura media delle superficie, "Rendic. dei Lincei », (6) 11 (1930), 50็4-558. 
It is known that the normal curvature $N$ of a curve in $F_{n-1}$ at a point $P$ with the tangent vector $t^{i}$ is given by

$$
N=n^{i} \frac{D t_{i}}{d s}=-t_{i} \frac{D n^{i}}{d s}=\Omega_{\lambda \mu} t^{\lambda} t^{\mu}
$$

where $D$ denotes covariant differentiation, $s$ is the arc-length of the curve and $n^{i}$ is the normal vector of $F_{n-1}$. Then the mean curvature $M$ of $F_{n-1}$ at $P$ is

$$
M=g^{\lambda \mu} \mathbf{Q}_{\lambda \mu} \mathbf{Q}_{\lambda}^{\lambda}
$$

In particular, when the direction of the element of support $(x, x)$ coincides with $n^{i}$ or with the tangential direction of $F_{n-1}$, we obtain the mean curvature of CARTAN $\left({ }^{3}\right)$ or that of BERWALD $\left({ }^{4}\right)$ respectively.

Consider in $F_{n-1}$ a variable closed hypersurface $F_{n-2}$ containing $P$ and denote by $w^{i}(Q)$ the contravariant components of the normal unit vector of $F_{n-2}$ in $F_{n-1}$ at a generic point $Q$ of $F_{n-2}$. If $w^{i}(Q / P)$ are the components of the vector obtained from $w^{i}(Q)$ by a parallel displacement with respect to $F_{n}$ from $Q$ to $P$, along an arbitrary arc $C$ in $F_{n-1}$, and the complementary of the angle between $w^{i}(Q / P)$ and $n^{i}(P)$ is taken as the angle $\theta$ between $w^{i}(Q)$ and the tangential hyperplane $E_{n-1}$ of $F_{n-1}$ at $P$, then we have the following theorem :

THEOREM 1. - The mean curvature $\mathrm{M}$ of $\mathrm{F}_{\mathrm{n}_{-1}}$ at $\mathrm{P}$ is given by

$$
M=\lim _{F_{n \rightarrow 2} \rightarrow P} \frac{1 \theta d \tau_{n-2}}{\int_{F_{n-1}}^{F_{n-1}} d \tau_{n-1}}
$$

where $\mathrm{R}_{\mathrm{n}-1}$ denotes the domain of $\mathrm{F}_{\mathrm{n}-1}$ enclosed by $\mathrm{F}_{\mathrm{n}-2}$, and $\mathrm{d} \tau_{\mathrm{n}-1}$ and $\mathrm{d} \tau_{\mathrm{n}-2}$ denote the volume elements of $\mathrm{R}_{n-1}$ and $\mathrm{F}_{\mathrm{n}-2}$ respectively.

Proof. - Without loss of generality, we can assume that the coordinates of $P$ and $Q$ in $F_{n-1}$ are $(0,0, \ldots, 0)$ and $\left(u^{1}, u^{2}, \ldots, u^{n-1}\right)$ respectively. Denoting the infinitesimal vector on $C$ by $\delta x^{i}=q_{i}^{i} \delta u^{\lambda}$, oriented by the sense

(3) E. Cartan, Les espace de Finsler, "Actualités scientifiques et industrielles", 79 (1934), Hermann Cie., Paris.

(4) L. BERWALD, Über die Kauptkrümmungen einer Fläche im dreidimensionalen Finslerschen Raum, * Monatsh. f. Math. n. Phys. ", 43 (1936), 1-14. 
from $Q$ to $P$, we have

$$
\begin{aligned}
w_{i}(Q / P)= & w_{i}(Q)-\int_{Q}^{P} \Gamma_{i k f}^{j}\left(x, x^{\prime}\right) w_{j}\left(x, x^{\prime}\right) \delta x^{k} \\
& -\int_{Q}^{P} A_{i k}^{j}\left(x, x^{\prime}\right) w_{j}\left(x, x^{\prime}\right) \omega^{k} \\
= & w_{i}(P)-\int_{Q}^{P} \frac{\partial w_{i}}{\partial x^{k}} \delta x^{k}-\int_{Q}^{P} \Gamma_{i h}^{*}\left(x, x^{\prime}\right) w_{j}\left(x, x^{\prime}\right) \delta x^{k} \\
& -\int_{Q}^{P} A_{i k}^{j}\left(x, x^{\prime}\right) w_{j}\left(x, x^{\prime}\right) \omega^{k} \\
= & w_{i}(P)+\int_{Q}^{P} D w_{i}\left(x, x^{\prime}\right),
\end{aligned}
$$

where we have placed

$$
w_{i}(P)=w_{i}(Q)-\int_{Q}^{P} \frac{\partial w_{i}}{\partial x^{k}} \delta x^{k}, \quad \omega^{h}=D l^{k} .
$$

Since $F_{n}$ is of a Euclidean connection, $w_{i}(Q / P)$ remain the covariant components of a unit vector. Hence from (3) it follows that

$$
\sin \theta=n^{i}(P) w_{i}(Q / P)=n^{i}(P)\left\{w_{i}(P)+\int_{Q}^{P} D w_{i}\right\} .
$$

Moreover, $w_{i}(P)$ is a covariant vector in $F_{n-1}$, so that

$$
n^{i}(P) w^{i}(P)=0, \quad n^{i}(P) D w_{i}(P)=-w_{i}(P) D n^{i}(P) .
$$

From (1) it is clear that

and consequently

$$
-\delta x^{i} D n_{i}=\Omega_{\lambda \mu} \delta u^{\lambda} \delta u^{\mu}=\mathbf{\Omega}_{\lambda \mu} \delta u^{\lambda} \delta x^{i} \hat{\vartheta}_{i}^{\mu},
$$

so we get from (4)

$$
-D n_{i}=\Omega_{\lambda \mu} \delta u^{\lambda} \vartheta_{i}^{\mu}
$$

$$
\begin{aligned}
\sin \theta & =\int_{Q}^{P} n^{i}(P) D w_{i}=\int_{Q}^{P} n^{i} D w_{i}+O\left(u^{\lambda}\right) \\
& =-\int_{Q}^{P} w_{i} D n^{i}+0\left(u^{\lambda}\right)=\int_{Q}^{P} \cdot Q_{\mu}^{\lambda} w_{\lambda} \delta u^{\mu}+O\left(u^{\lambda}\right) \\
& =Q_{\mu}^{\lambda}(Q) w_{\lambda}(Q) u^{\mu}+O\left(u^{\lambda}\right) \quad\left(u^{\lambda} \rightarrow 0\right) .
\end{aligned}
$$


Now suppose that $F_{n-2}$ is defined by

then

$$
u^{\lambda}=u^{\lambda}\left(v^{\alpha}\right) \quad(\alpha=1,2, \ldots, n-2) ;
$$

$$
d \tau_{n-2}=\sqrt{\bar{g}} d v^{1} d v^{2} \ldots d v^{n-9}
$$

where

$$
\bar{g}=\operatorname{det}\left(g_{\alpha \beta}\right), \quad g_{\alpha \beta}=g_{\lambda \mu} \frac{\partial u^{\lambda}}{\partial v^{\alpha}} \frac{\partial u^{\mu}}{\partial v^{\beta}} \quad(\alpha, \beta=1,2, \ldots, n-2)
$$

By changing the coordinates

$$
{ }_{\lambda}^{\lambda}=v^{\alpha}, \quad \underset{\lambda}{u^{\lambda}}=(-1)^{\lambda-1} u^{\lambda}, \quad \underset{\lambda}{u^{\beta}=v^{\beta-1} \quad(\alpha<\lambda<\beta),}
$$

$\lambda$ being not summed, we get

$$
\begin{aligned}
& w_{\lambda}=w_{\nu} \frac{\partial u^{\nu}}{\partial u_{\alpha}}=w_{\nu} \frac{\partial u^{\nu}}{\partial v^{\alpha}}=0, \\
& w_{\lambda}=w_{\nu} \frac{\partial u^{\nu}}{\partial u_{\lambda}^{\beta}}=w_{\nu} \frac{\partial u^{\nu}}{\partial v^{\beta-1}}=0,
\end{aligned}
$$

since $\frac{\partial u^{\nu}}{\partial v^{\alpha}}$ are the contravariant components of a vector in $F_{n-2}$, and $w_{\nu}$ the covariant components of a normal unit vector to $F_{n-2}$. Thus we are led to the relation

$$
{\underset{\lambda}{\lambda}}_{\lambda}=\frac{1}{\sqrt{g_{\lambda}}}=\frac{\sqrt{\bar{g}}}{\sqrt{\lambda}}
$$

$(\lambda$ not summed),

where $g_{\lambda}^{\mu \nu,}, \bar{g}$ and $\underset{\lambda}{g}$ denote the transformed expression of $g^{\mu, \nu}, \bar{g}$ and $g=\operatorname{det}\left(g_{\lambda \mu}\right)$ respectively, as it is easily seen from the assumption

$$
\underset{\lambda}{g_{\lambda}^{\mu \nu} w_{\mu} w_{\lambda}}=g_{\lambda}^{\lambda{ }_{\lambda} w_{\lambda}^{2}}=1
$$

$(\lambda$ not summed).

Therefore from (6), (7) and (8) we deduce

$$
\begin{aligned}
& w_{\lambda} d \tau_{\eta_{-2}}=w_{\lambda} \sqrt{\bar{g}}_{\bar{g}} d v^{1} d v^{2} \ldots d v^{n-2} \\
& =(-1)^{\lambda-1} w_{\lambda} \underbrace{}_{\lambda} \bar{g}_{\lambda} d u_{\lambda}^{1} d u^{2} \ldots d u_{\lambda}^{\lambda-1} d u_{\lambda}^{\lambda+1} \ldots d u_{\lambda}^{n-1} \\
& =(-1)^{\lambda-1} \underset{\lambda}{\sqrt{g}} d u_{\lambda}^{1} d u_{\lambda}^{2} \ldots d u_{\lambda}^{\lambda-1} d u_{\lambda}^{\lambda+1} \ldots d u^{n-1} \text {. }
\end{aligned}
$$


By applying the generalized GREEN's integral theorem, we obtain from (5) and (9) that

$$
\begin{aligned}
& \int_{F_{n-2}} \theta d \tau_{n-2}=\int_{F_{n-2}} \mathbf{Q}_{\mu}^{\lambda} u^{\mu} w_{\lambda} d \tau_{n-2}+O\left(u^{\lambda^{n-1}}\right) \\
& =\int_{F_{n=2}}^{n-1} \sum_{\lambda=1}^{\lambda} Q_{\mu}^{\mu} u_{\lambda} \sqrt{g}(-1)^{\lambda-1} d u_{\lambda}^{1} d u_{\lambda}^{2} \ldots d u_{\lambda}^{\lambda-1} d u_{\lambda}^{\lambda+1} \ldots d u_{\lambda}^{n-1}+0\left(u^{\lambda^{n-1}}\right) \\
& =\sum_{\lambda=1}^{n-1} \int_{\Gamma_{n-2}} \Omega_{\mu}^{\lambda} u^{\mu} \sqrt{g} \underset{\lambda}{g} d u_{\lambda}^{1} d u_{\lambda}^{2} \ldots d u_{\lambda}^{n-2}-\underset{\lambda}{d u^{1}} d u_{\lambda}^{2} \ldots \lambda_{\lambda} d u_{\lambda}^{n-3} d u^{n-1} \\
& +\ldots+(-1)^{\lambda-1} d u_{\lambda}^{1} d u_{\lambda}^{2} \ldots d u_{\lambda}^{\lambda-1} d u_{\lambda}^{\lambda+1} \ldots d u^{n-1} \\
& \left.+\ldots+(-1)^{n-2} d u_{\lambda}^{2} d u_{\lambda}^{3} \ldots d u_{\lambda}^{n-1}\right)+O\left(u^{\lambda^{n-1}}\right) \\
& =\sum_{\lambda=1}^{n-1} \int_{R_{n-1}} \mathbf{Q}_{\lambda}^{\lambda} d \tau_{n-i}+0\left(\boldsymbol{u}^{\lambda-1}\right) \\
& =\Omega_{\lambda}^{\lambda}(P) \int_{R_{n-1}} d \tau_{n-1}+0\left(u^{\lambda^{n-1}}\right), \\
& u^{\lambda}-0
\end{aligned}
$$

Thus we have completed the proof.

2. Mean eurvatures of an $m$-dimensional subspace $F_{m}$ in $F_{n}$.

There is $\mathbf{n}$. difficulty in extending the same arguments to the general case where an $m$-dimensional subspace $F_{m}$ is considered instead of $F_{n \cdot 1}$. For a normal vector $\vec{n}_{\sigma 1}$ to $F_{m}$, we can similarly define the mean curvature $M_{\sigma}$ of $F_{m}$ at a point $P$. Denote, in fact, by $F_{m-1}$ a variable closed hypersur. face in $F_{m}$ containing $P$ and by $\theta_{\sigma}$ the angle between the normal vector $w_{i}$ to $F_{m}$, in $F_{m}$ and the tangential $(n-1)$-dimensional affine space $E_{\sigma t, n-1}$ orthogonal to $\vec{n}_{\sigma 1}$. Then we have

Theorem 2. - The mean curvature $\mathrm{M}_{\sigma}$ of $\mathrm{F}_{\mathrm{m}}$ at $\mathrm{P}$ corresponding to $\overrightarrow{\mathrm{n}}_{\sigma \mathrm{t}}$ is given by

$$
M_{\sigma}=\lim _{F_{m-1} \rightarrow P} \frac{\int_{F_{m}-1}^{\theta_{\sigma} d \tau_{m-1}}}{\int_{R_{m}} d \tau_{m}},
$$

where $\mathrm{R}_{\mathrm{m}}$ denotes the domain of $\mathrm{F}_{\mathrm{m}}$ enclosed by $\mathrm{F}_{\mathrm{m}-1}$, and $\mathrm{d} \tau_{\mathrm{m}}$ and $\mathrm{d} \tau_{\mathrm{m}-1}$ denote the volume elements of $\mathrm{R}_{\mathrm{m}}$ and $\mathrm{F}_{\mathrm{m}-1}$ respectively.

Coroltary 1. - If, in particular, $F_{m}$ is "gespannt", i. e., if the $(n-m)$-dimensional affine space $E_{n}^{n-m}$ orthogonal to $E_{m}$ at a point $P$ of $F_{m}$ can be determined uniquely, then there exists a normal vector $\vec{n}$, which we 
shall call the mean curvature normal, such that the mean curvature $M$ of $F_{m}$ at $P$ for $\vec{n}$ is given by the equation

$$
M=\lim _{F_{m-1} \rightarrow P} \frac{\int_{F_{m-1}}^{\theta} \theta d \tau_{m-1}}{\int_{R_{m}} d \tau_{m}}=\sqrt{\sum_{\sigma=m+1}^{n} M_{\sigma}^{2}}
$$

where $\theta$ denotes the angle between $w_{i}$ and the tangential affine space $E_{n-1}$ orthogonal to $\vec{n}$, and the components of $\vec{n}$ are

$$
n^{i}=\sum_{\sigma=m+1}^{n} \mathbf{\Omega}_{\sigma 1 \lambda \mu} g^{\lambda \mu} n_{\sigma 1}{ }^{i}
$$

independent of the choice of the orthogonal ennuple $\left\{\vec{n}_{a_{i}}\right\}$, so that $\vec{n}$ has the length $M$.

We shall call $M$ the mean curvature of the "gespannt subspace $F_{m}$. Corollary 2. - If $F_{m}$ is "gespannt》, then its mean curvature is zero if its mean curvature for any normal vector is zero.

Corollary 3. - The mean curvature of a "gespannt» subspace $F_{m}$, for any normal vector orthogonal to the mean curvature normal, is zero.

These corollaries are evident generalizations of some known results in Riemannian geometry $(5)$.

The author wishes to express his thanks to Prof. B. Su for the suggestions during the preparation of this paper.

(5) Cf, for example, L. P. Eisenhart, Riemannian geometry, "Princeton Univ. Press. ", (1925), 169.170. 